\title{
Resting state amygdala-prefrontal connectivity predicts symptom change after cognitive behavioral therapy in generalized social anxiety disorder
}

Heide Klumpp ${ }^{1,2,3^{*}}$, Michael K Keutmann ${ }^{2}$, Daniel A Fitzgerald ${ }^{1,4}$, Stewart A Shankman ${ }^{2,3}$ and K Luan Phan ${ }^{1,2,3,4}$

\begin{abstract}
Background: Aberrant amygdala-prefrontal interactions at rest and during emotion processing are implicated in the pathophysiology of generalized social anxiety disorder (gSAD), a common disorder characterized by fears of potential scrutiny. Cognitive behavioral therapy (CBT) is first-line psychotherapy for gSAD and other anxiety disorders. While CBT is generally effective, there is a great deal of heterogeneity in treatment response. To date, predictors of success in CBT for gSAD include reduced amygdala reactivity and increased activity in prefrontal regulatory regions (e.g., anterior cingulate cortex, ACC ) during emotion processing. However, studies have not examined whether tonic (i.e., at rest) coupling of amygdala and these prefrontal regions also predict response to CBT.
\end{abstract}

Results: Twenty-one patients with gSAD participated in resting-state functional magnetic resonance imaging (fMRl) before 12 weeks of CBT. Overall, symptom severity was significantly reduced after completing CBT; however, the patients varied considerably in degree of symptom change. Whole-brain voxel-wise findings showed symptom improvement after CBT was predicted by greater right amygdala-pregenual ACC ( pgACC ) connectivity and greater left amygdala-pgACC coupling encompassing medial prefrontal cortex. In support of their predictive value, area under receiver operating characteristic curve was significant for the left and right amygdala-pgACC in relation to treatment responders.

Conclusions: Improvement after CBT was predicted by enhanced resting-state bilateral amygdala-prefrontal coupling in GSAD. Preliminary results suggest baseline individual differences in a fundamental circuitry that may underlie emotion regulation contributed to variation in symptom change after CBT. Findings offer a new approach towards using a biological measure to foretell who will most likely benefit from CBT. In particular, the departure from neural predictors based on illness-relevant stimuli (e.g., socio-emotional stimuli in gSAD) permits the development of biomarkers that reflect commonalities in the neurobiology of anxiety and mood disorders.

Keywords: Generalized social anxiety, fMRI, Treatment, Brain imaging, Rest

\footnotetext{
* Correspondence: hklumpp@psych.uic.edu

${ }^{1}$ Mood and Anxiety Disorders Research Program, Department of Psychiatry

(HK, DAF, KLP), University of Illinois at Chicago, 1747 W. Roosevelt Rd,

Chicago, IL 60608, USA

${ }^{2}$ Department of Psychology (HK, MKK, SAS, KLP), University of Illinois at

Chicago, Chicago, IL, USA

Full list of author information is available at the end of the article
}

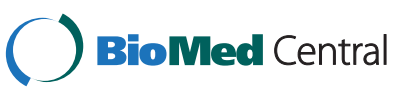

2014 Klumpp et al.; licensee BioMed Central. This is an Open Access article distributed under the terms of the Creative Commons Attribution License (http://creativecommons.org/licenses/by/4.0), which permits unrestricted use, distribution, and reproduction in any medium, provided the original work is properly credited. The Creative Commons Public Domain Dedication waiver (http://creativecommons.org/publicdomain/zero/1.0/) applies to the data made available in this article unless otherwise stated. 


\section{Background}

Cognitive behavioral therapy (CBT) is empirically supported psychotherapy for generalized social anxiety disorder (gSAD), a common, debilitating illness marked by excessive fears of negative evaluation by others [1]. CBT primarily attempts to reduce symptoms via cognitive restructuring, an emotion regulation strategy aimed at decreasing thought-related negative affect, in conjunction with exposure exercises (e.g., facing anxiety-evoking situations). While generally effective, treatment response is varied with approximately $30 \% 40 \%$ of patients with gSAD not fully responding to CBT $[2,3]$. Findings from neuroimaging studies indicate heterogeneity in treatment outcome may relate in part to brain regions implicated in the pathophysiology of gSAD that are utilized by CBT.

Accumulating data indicate the amygdala, a key emotion processing region that mediates fear [4], plays a prominent role in gSAD. The amygdala has interconnections to prefrontal regions that down-regulate emotional reactivity (e.g., medial prefrontal cortex (mPFC); [5]). In gSAD, amygdala hyper-reactivity to salient signals has been demonstrated in addition to disturbances in regulatory regions (e.g., exaggerated or attenuated mPFC activation; [6]). Moreover, in the absence of stimuli presentation or task engagement (i.e., during rest), aberrant amygdala connectivity with prefrontal regulatory areas (e.g., anterior cingulate cortex (ACC), medial orbitofrontal cortex (mOFC)) has been observed [7-9]. Findings suggest phasic hyper-reactive amygdala responses to external information involve tonic disturbances in core amygdala-prefrontal circuitry [8] and that individual differences in such circuitry may factor into the likelihood of benefiting from CBT.

To date, studies of amygdala as a brain-based marker in predicting CBT response in gSAD appear to be limited to emotion perception tasks, and results have been mixed. For example, we recently showed less pre-CBT amygdala activity to emotional faces predicted CBT success [10]; however, other emotion processing studies of gSAD have not revealed amygdala effects [11,12]. Regarding prefrontal regions as predictors, we have observed a positive link between dorsal ACC and mOFC activity in gSAD during emotion processing and symptom improvement in CBT $[10,12]$ even in the absence of amygdala findings [12]. However, it is not clear whether amygdala response contributed to symptom change as regions were examined in isolation as opposed to nodes in a network.

A means of increasing our understanding of amygdalabased circuitry as a biomarker in predicting who will likely respond to $\mathrm{CBT}$ is with resting-state functional MRI (rs-fMRI). An advantage of rs-fMRI is that it examines fundamental networks that are task independent but may underlie emotion and regulatory processes in the unprovoked state [13]. Therefore, the objective of this study was to use pre-CBT rs-fMRI to investigate the relationship between amygdala-prefrontal coupling and CBT success in gSAD. Based on the literature [10,12], we hypothesized greater rs-fMRI amygdala-ACC or amygdalamOFC connectivity would correspond with $\mathrm{CBT}$ response.

\section{Methods}

\section{Participants}

All 21 participants (14 female, 7 male) with an average age of 28.3 9.4 years met criteria for gSAD based on the Structured Clinical Interview for DSM-IV (SCID) [14]. Symptom severity was assessed with the Liebowitz Social Anxiety Scale (LSAS) [15] administered by licensed clinicians, and depression level was measured with the Beck Depression Inventory [16]. Clinical Global Impression-Improvement (CGI-I; [17]), comprising a 7point scale $(1=$ very much improved, $7=$ worsening symptoms), was used to determine whether or not a patient responded to treatment.

All the participants were free of psychotropic medication, except for two who were on a stable dose of bupropion for at least 8 weeks prior to, and throughout, the study. Exclusion criteria included current or recent (within 6 months of study) comorbid major depressive disorder or recent substance abuse/dependence or any history of major psychiatric illness (e.g., bipolar, psychotic disorder).

The participants were between 18 and 55 years of age, right-handed, and free of current and past major medical or neurologic illness, as confirmed by a Board Certified physician. None of the participants tested positive for alcohol or illegal substances. The study protocol was approved by the Institutional Review Boards of the University of Michigan Medical School, and as per protocol, all the participants provided written informed consent.

The patients received 12 weeks of manualized individual CBT conducted by the same doctoral-level licensed clinical psychologist who has several years of training in CBT. A licensed clinical psychologist with both expertise in $\mathrm{CBT}$ and clinical trial investigations involving CBT provided supervision to ensure adherence to treatment. CBT encompassed psychoeducation, cognitive restructuring, in vivo exposures, and relapse prevention [18].

\section{Resting-state fMRI}

Padding with foam cushions was used to reduce head movement. The participants were instructed to fixate on a crosshair centrally displayed on the blank gray screen, relax, and let their mind wander without falling asleep for $8 \mathrm{~min}$.

\section{Functional imaging: acquisition and analysis}

Magnetic resonance imaging (MRI) was performed on a 3 T GE Signa System (Milwaukee, WI) acquiring bloodoxygen-level-dependent (BOLD) images with a T2*sensitive gradient-echo reverse spiral acquisition $(3 \mathrm{~mm}$ 


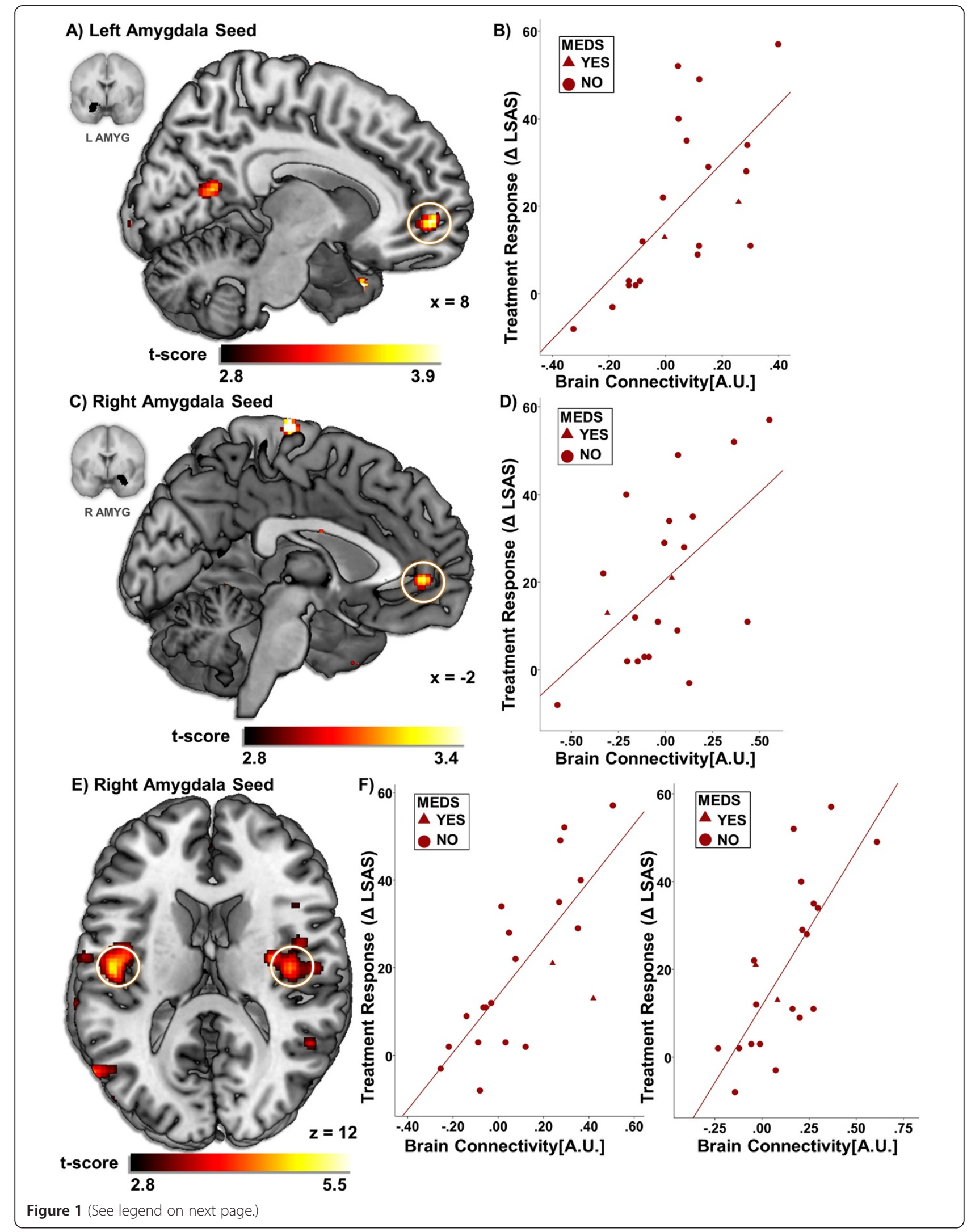


(See figure on previous page.)

Figure 1 Regressing LSAS change and scatterplot of regression analyses. (A) Regressing LSAS change $\left(\Delta_{\text {PreTx-PostTx }}\right)$ while initial severity (LSAS PreTx) is controlled for as a regressor of no interest; brain map depicts whole-brain analysis of covariance showing enhanced left amygdala-anterior cingulate cortex/medial prefrontal cortex coupling during rest in gSAD displayed on statistical t-map at $p<0.005$. (B) Scatterplot of regression analyses depicting extracted measures of left amygdala-anterior cingulate cortex/medial prefrontal cortex connectivity and relation to change in social anxiety severity. (C) Regressing LSAS change $\left(\Delta_{\text {PreTx-PostTx }}\right)$ while baseline severity $\left(\right.$ LSAS PreTx $\left._{\text {re }}\right)$ is controlled for as a regressor of no interest; brain map depicts whole-brain analysis of covariance showing enhanced right amygdala-anterior cingulate cortex connectivity during rest in GSAD displayed on statistical t-map at $p<0.005$. (D) Scatterplot of regression analyses depicting extracted measures of right amygdala-anterior cingulate cortex coupling and relation to change in social anxiety severity. (E) Regressing LSAS change $\left(\Delta_{\text {PreTX-PostTx }}\right)$ while initial severity (LSAS PreTx $)$ is controlled for as a regressor of no interest; brain map depicts whole-brain analysis of covariance showing enhanced right amygdala-bilateral insula connectivity during rest in $\mathrm{gSAD}$ displayed on statistical t-map at $p<0.005$. (F) Scatterplot of regression analyses illustrating extracted measures of right amygdala-bilateral insula coupling and relation to change in social anxiety severity. LSAS, Liebowitz Social Anxiety Scale; CBT, cognitive behavioral therapy; gSAD, generalized social anxiety disorder.

43 axial slices; 2 s TR; 30 ms TE; 6464 matrix; 220 mm FOV; 90 flip) optimized to minimize susceptibility artifacts in the medial temporal pole. High-resolution, T1weighted anatomical scans (3D-SPGR; $9 \mathrm{~ms}$ TR; $1.8 \mathrm{~ms}$ TE; 15 flip; 256256 matrix; 256 mm FOV, 1.2 mm 124 axial slices) were also acquired for precise anatomical localization and normalization.

Analyses were performed using the Functional Connectivity (CONN) toolbox [19], which employs routines from the Statistical Parametric Mapping software (SPM8; Wellcome Trust Centre for Neuroimaging, London, UK). Eight initial volumes from each resting-state run were discarded to allow for T1 equilibration effects. Images were realigned to correct for motion, corrected for errors in slice timing, spatially transformed to standard MNI space using the functional template provided with SPM8, resampled to 2-mm voxels, and smoothed with an 8-mm FWHM Gaussian kernel prior to statistical analysis. The participants had no movement greater than 2-mm translation or 2 rotation across the run. Effects of nuisance variables (global, white matter and CSF signals and movement parameters) were reduced following the CompCor strategy [20]; data were band-pass filtered to $0.010 .09 \mathrm{~Hz}$.

Temporal correlations of the resting-state BOLD signal time series were examined between the left and right amygdala seed regions (anatomically derived regions of interest from the Automated Anatomical Labeling (AAL) toolbox [21]) and the rest of the brain. During second-level processing, LSAS change $\left(\Delta_{\text {PreTx-PostTx }}\right)$ was regressed with initial severity $\left(\mathrm{LSAS}_{\mathrm{PreTx}}\right)$ controlled for as a regressor of no interest. The ACC and medial OFC regions of interest were examined at the whole-brain level with significance defined as $p<0.005$ uncorrected with more than 20 contiguous voxels per cluster $\left(>160\right.$ volume $\left.\mathrm{mm}^{3}\right)$ to strike a balance between type I and II errors [22]. The AAL atlas $[21,23]$ was used to identify regions of interest (ROIs) and other significant whole-brain findings across subjects.

To clarify the directionality and magnitude of baseline amygdala-prefrontal connectivity related to change in symptom severity, 10-mm-diameter spherical ROIs were generated around the peak activation of a whole-brain cluster. Subsequently, parameter estimates $(\beta$ weights and arbitrary units (au)) were extracted from the ROIs for each participant and submitted to Pearson s correlations and scatterplots in the Statistical Package for the Social Sciences (SPSS version 20; Chicago, IL). Additionally, the parameter estimates were used to calculate the area under a receiver operating characteristic (ROC) curve in SPSS to assess the predictive value of a priori connectivity results in terms of CBT responders based on CGI-I. Apart from fMRI, we performed a regression analysis in SPSS to examine whether demographic factors (i.e., age, gender, education level) independently effected LSAS change $\left(\Delta_{\text {PreTx-PostTx }}\right)$.

\section{Results}

\section{Treatment effects on social anxiety}

Symptom severity assessed by LSAS significantly decreased from an average of 71.611 .9 to 51.519 .5 ( $t=$ 4.87, $p<0.001$ ). The clinical cutoff is $\geq 60$ for gSAD [24]; therefore, results point to a significant overall improvement with variation in degree of symptom change. Additionally, depression level which was in the minimal range [16] at the start of CBT (11.7 8.3) significantly decreased (5.0 6.0) $(t=4.60, p<0.001)$. Based on the CGI-I, about $70 \%$ of the patients with gSAD (15 of 21) were responders as they were rated to be very much improved or much improved (CGI-I score of 1 or 2) whereas 6 patients had a CGI-I score of $>2$ posttreatment and were thus considered non-responders. Regression analysis findings were not significant for age, gender, or education level (all $p s>0.05$ ).

\section{fMRI}

For the right amygdala, LSAS change $\left(\Delta_{\text {PreTx-PostTx }}\right)$ was predicted by more baseline connectivity with the left pregenual ACC ( pgACC ) (i.e., anterior cingulum) [(-4, $48,0), z=2.90$, volume $\left.=392 \mathrm{~mm}^{3} ; r=0.55, p<0.010\right]$ (Figure 1). Area under an ROC curve regarding the right amygdala-pgACC was 0.80 in the context of CBT 
responders which was significant $(p<0.04)$. Similar pgACC results were observed for the left amygdala [(10, $52,-2), z=3.30$, volume $\left.=928 \mathrm{~mm}^{3} ; r=0.66, p<0.001\right]$ though here the cluster extended to the medial prefrontal cortex (i.e., frontal medial orbital gyrus) volume $=$ $712 \mathrm{~mm}^{3}$ (Figure 1). Again, area under the curve (i.e., $0.83)$ was significant $(p<0.02)$. As to regions beyond a priori prefrontal areas, we observed symptom improvement robustly corresponded with bilateral insula (i.e., rolandic operculum) coupling [left: $(-36,-30,26)$, $z=4.00$, volume $=2,648 \mathrm{~mm}^{3} ; r=0.75, p<0.001$; right: (30, -10, 18), $z=4.14$, volume $=2,192 \mathrm{~mm}^{3} ; r=0.74$, $p<0.001]$ related to the right amygdala. Area under the curves concerning the right amygdala-left insula and right amygdala-right insula were significant (i.e., 0.84, $p<0.02 ; 0.80, p<0.04$, respectively) (Figure 1). For completeness, we report all results outside regions of interest in Table 1.

\section{Discussion}

As hypothesized, clinical improvement following CBT in the patients with gSAD was predicted by greater pretreatment amygdala connectivity with prefrontal regions implicated in controlling emotion. Specifically, greater symptom reduction was foretold by increased pre-CBT right amygdala-pgACC and left amygdala-pgACC/mPFC coupling, a circuit involved in emotion processing and regulation $[25,26]$. In support of its predictive capacity, ROC results pertaining to $\mathrm{CBT}$ responder based on a CGI-I cutoff were also significant. Pointing to the potential relevance of the circuit as a brain predictor and/ or target for treatment is a resting-state study showing lower amygdala-ACC/mPFC connectivity in gSAD correlated with social anxiety severity and that deficient coupling was enhanced by an acute challenge of the neuropeptide oxytocin [9]. Together, findings indicate intrinsic amygdala-medial prefrontal interactions may play a role in predicting the likelihood of responding to an intervention in gSAD. Findings expand on emotion activation paradigms that have demonstrated associations between increases in ACC or mOFC activity before treatment and improvement after CBT in gSAD $[10,12]$. Further study is needed to examine whether phasic (e.g., task/emotion-based) in combination with tonic (i.e., at rest ) biomarkers can be used to predict response to CBT.

Beyond prefrontal regions of interest, symptom change was foretold by more and less connectivity in an extensive network indicative of the regions interconnected with the amygdala (e.g., insula, occipital lobe, middle temporal gyrus, superior frontal gyrus, parahippocampal gyrus; [27]) in addition to wide-scale coupling within and between networks exhibited at rest [13]. We did not have $a$ priori hypotheses for these regions and, therefore, hesitate to interpret these preliminary, exploratory findings. Nevertheless, it is interesting to note symptom improvement also positively corresponded with the right amygdala-insula (i.e., rolandic operculum) coupling and, based on ROC findings, served as a good estimate of treatment response. The insula is proposed to play a role in anxiety disorders [28,29], which is supported by observations of exaggerated insula reactivity to emotional stimuli in gSAD relative to healthy controls [6]. In the context of treatment for gSAD, we observed insula hyper-reactivity to threat relevant stimuli decreased after CBT [12]; however, task-based pre-CBT insula activity to threat has not yet been shown to predict symptom change in gSAD [10-12]. Our findings suggest that in the absence of external stimuli, baseline emotion processing circuitry appears to function as a predictor. More study is needed to understand how the intrinsic amygdala-insula and other resting-state networks beyond a priori regions might be utilized by CBT.

\section{Conclusions}

First, our study is not without important limitations. These include a relatively small sample size which increases risk for type II errors. Second, 2 of the 21 participants with gSAD were taking bupropion. Even though the medication was stable before the study and remained unchanged during the study, and these participants did not serve as outliers in a priori findings as indicated by scatterplots, any influence it may have had on other outcomes cannot be ruled out. Third, the lack of a waitlist group to serve as a control for changes in symptoms unrelated to treatment reduces our ability to draw firm conclusions about neural predictors of CBT response. Fourth, replication in an independent sample is necessary before conclusions can be made as to the clinical relevance of our findings. Fifth, connectivity results were limited to the bilateral amygdala. Future studies may want to seed prefrontal regions implicated in emotion regulation (e.g., dorsolateral, dorsomedial prefrontal cortex; orbitofrontal cortex; anterior cingulate cortex; [5]) to examine their relationship with the amygdala and ability to predict CBT success. Sixth, the lack of independent evaluators of treatment fidelity and symptom change warrants replication and further investigation. Despite limitations, findings suggest individual differences in intrinsic amygdala-prefrontal connectivity can help explain the heterogeneity in response to $\mathrm{CBT}$ in gSAD. Findings also indicate resting-state fMRI may be a useful approach in identifying brain-based biomarkers in treatment response. Among the advantages of resting-state biomarkers is the ease of application across other internalizing psychopathologies that may have common pathophysiology and for which CBT is an empirically validated treatment option (e.g., post-traumatic stress disorder, major depressive disorder). 
Table 1 Whole-brain voxel-wise regression: relation between pre-treatment to post-treatment change in social anxiety severity, controlling for pre-treatment severity

\begin{tabular}{|c|c|c|c|c|c|c|}
\hline & & MNI & & & Volume & \\
\hline & Region & $x$ & $y$ & $z$ & $\left(\mathrm{~mm}^{3}\right)$ & $z$ \\
\hline Right amygdala & & & & & & \\
\hline Positive correlation & $\mathrm{R}$ rolandic operculum & 30 & -10 & 18 & 2,192 & 4.14 \\
\hline & L rolandic operculum & -36 & -30 & 26 & 2,648 & 4.00 \\
\hline & $\mathrm{L}$ middle temporal gyrus & -58 & -66 & 12 & 800 & 3.67 \\
\hline & $\mathrm{L}$ angular gyrus & -36 & -70 & 48 & 552 & 3.38 \\
\hline & $\mathrm{R}$ frontal inferior triangularis & 48 & 26 & 10 & 760 & 3.26 \\
\hline & $\mathrm{L}$ middle temporal gyrus & -66 & -34 & 2 & 608 & 3.18 \\
\hline & L paracentral lobule & -8 & -20 & 78 & 344 & 3.15 \\
\hline & $\mathrm{R}$ middle frontal gyrus & 26 & 34 & 34 & 408 & 2.96 \\
\hline & $\mathrm{L}$ anterior cingulum & -4 & 48 & 0 & 392 & 2.90 \\
\hline Negative correlation & L parahippocampal gyrus & -14 & 2 & -18 & 528 & 4.01 \\
\hline & $L$ temporal pole superior gyrus & -26 & 14 & -26 & 440 & 4.00 \\
\hline & R hippocampus & 24 & -10 & -20 & 328 & 3.98 \\
\hline & R frontal superior orbital gyrus & 16 & 16 & -18 & 264 & 3.73 \\
\hline & R gyrus rectus & 6 & 32 & -18 & 760 & 3.69 \\
\hline & $\mathrm{R}$ frontal superior gyrus & 16 & 66 & 26 & 360 & 3.64 \\
\hline & $R$ frontal middle orbital gyrus & 38 & 42 & -10 & 512 & 3.64 \\
\hline & $\mathrm{R}$ frontal superior medial gyrus & 10 & 40 & 56 & 352 & 3.48 \\
\hline & $\mathrm{L}$ cerebellum & -4 & -40 & -18 & 336 & 3.33 \\
\hline & $L$ frontal middle orbital gyrus & -22 & 58 & -10 & 296 & 3.04 \\
\hline & $L$ fusiform gyrus & -32 & -18 & -18 & 360 & 2.18 \\
\hline Left amygdala & & & & & & \\
\hline Positive correlation & L calcarine gyrus & -12 & -60 & 16 & 1,136 & 4.44 \\
\hline & $\mathrm{R}$ temporal pole middle gyrus & 32 & 18 & -36 & 344 & 3.58 \\
\hline & $\mathrm{R}$ anterior cingulum & 10 & 52 & -2 & 928 & 3.30 \\
\hline & $\mathrm{R}$ frontal medial orbital gyrus & & & & 712 & 3.30 \\
\hline & L middle occipital gyrus & -38 & -78 & 20 & 344 & 3.27 \\
\hline & R calcarine gyrus & 8 & -58 & 14 & 464 & 3.06 \\
\hline Negative correlation & L caudate & -18 & -14 & 18 & 224 & 4.67 \\
\hline & $\mathrm{R}$ frontal middle orbital gyrus & 36 & 64 & -4 & 1,104 & 4.05 \\
\hline & $L$ frontal middle gyrus & -44 & 20 & 48 & 1,104 & 3.97 \\
\hline & $R$ inferior occipital gyrus & 36 & -64 & -8 & 472 & 3.87 \\
\hline & $\mathrm{L}$ cerebellum & -42 & -80 & -22 & 784 & 3.77 \\
\hline & $\mathrm{R}$ frontal superior medial gyrus & 6 & 42 & 52 & 1,744 & 3.74 \\
\hline & $R$ frontal superior gyrus & 14 & 68 & 24 & 576 & 3.68 \\
\hline & $\mathrm{R}$ cerebellum & 4 & -48 & -44 & 432 & 3.60 \\
\hline & R frontal superior orbital gyrus & 18 & 38 & -16 & 376 & 3.53 \\
\hline & $R$ frontal inferior orbital gyrus & 36 & 38 & -12 & 480 & 3.47 \\
\hline & $L$ frontal middle orbital gyrus & -22 & 52 & -10 & 360 & 3.36 \\
\hline & R superior parietal lobule & 20 & -62 & 60 & 440 & 3.21 \\
\hline & $\mathrm{L}$ cerebellum & -18 & -90 & -30 & 200 & 3.15 \\
\hline & $\mathrm{R}$ inferior temporal gyrus & 56 & -66 & -8 & 296 & 3.00 \\
\hline & $R$ frontal middle gyrus & 42 & 16 & 54 & 168 & 2.84 \\
\hline
\end{tabular}

All listed clusters are significant at $p<0.005$ (uncorrected) with a threshold of greater than 160 volume $\left(\mathrm{mm}^{3}\right)$. 


\section{Abbreviations}

CBT: cognitive behavioral therapy; gSAD: generalized social anxiety disorder; ACC: anterior cingulate cortex; mOFC: medial orbitofrontal cortex; rs-fMRI: resting-state functional magnetic resonance imaging; DSM-IV: diagnostic and statistical manual of mental disorders fourth edition; SCID: Structured Clinical Interview for DSM Disorders; LSAS: Liebowitz Social Anxiety Scale; BDI: Beck Depression Inventory; CGII-I: Clinical Global Impression-Improvement; BOLD: blood-oxygen-level-dependent; 3T: 3.0 Tesla; GE: general electric; T2: spin-spin relaxation time; TR: repetition time; TE: echo time; $\mathrm{ms}$ : millisecond; $\mathrm{mm}$ : millimeter; FOV: field of view; 3D-SPGR: three-dimensional spoiled gradient-recalled acquisition in steady state; FWHM: full width at half maximum; CSF: cerebrospinal fluid; CompCor: component-based noise correction method; Hz: Hertz; $\beta$ : beta; pgACC: pregenual anterior cingulate cortex; mPFC: medial prefrontal cortex; PreTx: pre-treatment; PostTx: post-treatment; AAL: automated anatomical labeling; ROI: region of interest; SPSS: Statistical Package for the Social Sciences; ROC: receiver operating characteristic; au: arbitrary units.

\section{Competing interests}

The authors declare that they have no competing interests.

\section{Authors contributions}

HK conceived and designed the study, conducted the statistical analysis, data interpretation, and wrote the manuscript. MKK and DAF conducted the neuroimaging analyses and participated in the data interpretation and drafting of the manuscript. SAS participated in the data interpretation and drafting of the manuscript. KLP conceived and designed the study, conducted the data interpretation, and participated in the drafting of the manuscript. All authors read and approved the final manuscript.

\section{Acknowledgements}

This work was supported by grants from the National Institutes of Health and National Institute of Mental Health (MH076198 to KLP and MH093679 to $H K)$.

\section{Author details}

${ }^{1}$ Mood and Anxiety Disorders Research Program, Department of Psychiatry (HK, DAF, KLP), University of Illinois at Chicago, 1747 W. Roosevelt Rd, Chicago, IL 60608, USA. '2Department of Psychology (HK, MKK, SAS, KLP), University of Illinois at Chicago, Chicago, IL, USA. ${ }^{3}$ Department of Psychiatry (HK, SAS, KLP), University of Illinois at Chicago, Chicago, IL, USA. ${ }^{4}$ Mental Health Service (DAF, KLP), Jesse Brown VA Medical Center, Chicago, IL, USA.

Received: 8 September 2014 Accepted: 18 November 2014

Published online: 09 December 2014

\section{References}

1. American Psychiatric Association: Diagnostic and Statistical Manual of Mental Disorders, Fourth Edition: DSM-IV-TR. Washington, D.C.: American Psychiatric Association; 2000.

2. Heimberg RG, Liebowitz MR, Hope DA, Schneier FR, Holt CS, Welkowitz LA, Juster HR, Campeas R, Bruch MA, Cloitre M, Fallon B, Klein DF: Cognitive behavioral group therapy vs phenelzine therapy for social phobia: 12-week outcome. Arch Gen Psychiatry 1998, 55:1133 1141.

3. Davidson JRT, Foa EB, Huppert JD, Keefe FJ, Franklin ME, Compton JS, Zhao N, Connor KM, Lynch TR, Gadde KM: Fluoxetine, comprehensive cognitive behavioral therapy, and placebo in generalized social phobia. Arch Gen Psychiatry 2004, 61:1005 1013.

4. LeDoux JE: Emotion circuits in the brain. Annu Rev Neurosci 2000, 23:155 184.

5. Banks SJ, Eddy KT, Angstadt M, Nathan PJ, Phan KL: Amygdala-frontal connectivity during emotion regulation. Soc Cogn Affect Neurosci 2007, 2:303 312.

6. Brhl AB, Delsignore A, Komossa K, Weidt S: Neuroimaging in social anxiety disorder a meta-analytic review resulting in a new neurofunctional model. Neurosci Biobehav Rev 2014, 47C:260 280.

7. Hahn A, Stein P, Windischberger C, Weissenbacher A, Spindelegger C, Mose E, Kasper S, Lanzenberger R: Reduced resting-state functional connectivity between amygdala and orbitofrontal cortex in social anxiety disorder. Neuroimage 2011, 56:881 889.

8. Prater KE, Hosanagar A, Klumpp H, Angstadt M, Phan KL: Aberrant amygdala-frontal cortex connectivity during perception of fearful faces and at rest in generalized social anxiety disorder. Depress Anxiety 2013, 30:234 241.

9. Dodhia S, Hosanagar A, Fitzgerald DA, Labuschagne I, Wood AG, Nathan PJ, Phan KL: Modulation of resting-state amygdala-frontal functional connectivity by oxytocin in generalized social anxiety disorder. Neuropsychopharmacol Off Publ Am Coll Neuropsychopharmacol 2014 39:2061 2069.

10. Klumpp H, Fitzgerald DA, Angstadt M, Post D, Phan KL: Neural response during attentional control and emotion processing predicts improvement after cognitive behavioral therapy in generalized social anxiety disorder. Psychol Med 2014, 44:3109 3121.

11. Doehrmann O, Ghosh SS, Polli FE, Reynolds GO, Horn F, Keshavan A, Triantafyllou C, Saygin ZM, Whitfield-Gabrieli S, Hofmann SG, Pollack M, Gabrieli JD: Predicting treatment response in social anxiety disorder from functional magnetic resonance imaging. JAMA Psychiatr 2013, 70:87 97.

12. Klumpp H, Fitzgerald DA, Phan KL: Neural predictors and mechanisms of cognitive behavioral therapy on threat processing in social anxiety disorder. Prog Neuropsychopharmacol Biol Psychiatry 2013, 45:83 91.

13. Fox MD, Snyder AZ, Vincent JL, Corbetta M, Van Essen DC, Raichle ME: The human brain is intrinsically organized into dynamic, anticorrelated functional networks. Proc Natl Acad Sci U S A 2005, 102:9673 9678.

14. First MB, Spitzer RL, Gibbon M, Williams JBW: Structured Clinical Interview for DSM-IV Axis I Disorders, Clinician Version (SCID-CV). Washington, D.C: American Psychiatric Association; 1996.

15. Liebowitz MR: Social phobia. Mod Probl Pharmacopsychiatry 1987, 22:141 173

16. Beck AT, Steer RA, Ball R, Ranieri WF: Comparison of beck depression inventories-IA and-II in psychiatric outpatients. J Pers Assess 1996, 67:588 597.

17. Busner J, Targum SD: The clinical global impressions scale. Psychiatry 2007, 4:28 37

18. Hope DA, Heimberg RG, Turk CL: Managing Social Anxiety: A Cognitive-Behavioral Therapy Approach. 1st edition. New York, USA: Oxford University Press; 2006.

19. Whitfield-Gabrieli S, Nieto-Castanon A: Conn: a functional connectivity toolbox for correlated and anticorrelated brain networks. Brain Connect 2012, 2:125 141

20. Behzadi Y, Restom K, Liau J, Liu TT: A component based noise correction method (CompCor) for BOLD and perfusion based fMRI. Neuroimage 2007, 37:90 101

21. Tzourio-Mazoyer N, Landeau B, Papathanassiou D, Crivello F, Etard O, Delcroix N, Mazoyer B, Joliot M: Automated anatomical labeling of activations in SPM using a macroscopic anatomical parcellation of the MNI MRI single-subject brain. Neuroimage 2002, 15:273 289.

22. Lieberman MD, Cunningham WA: Type I and type II error concerns in fMRI research: re-balancing the scale. Soc Cogn Affect Neurosci 2009, 4:423 428

23. Lancaster JL, Woldorff MG, Parsons LM, Liotti M, Freitas CS, Rainey L, Kochunov PV, Nickerson D, Mikiten SA, Fox PT: Automated Talairach atlas labels for functional brain mapping. Hum Brain Mapp 2000, 10:120 131

24. Mennin DS, Fresco DM, Heimberg RG, Schneier FR, Davies SO, Liebowitz MR: Screening for social anxiety disorder in the clinical setting: using the Liebowitz Social Anxiety Scale. J Anxiety Disord 2002, 16:661 673.

25. Etkin A, Egner $T$, Kalisch R: Emotional processing in anterior cingulate and medial prefrontal cortex. Trends Cogn Sci 2011, 15:85 93

26. Ongr D, Ferry AT, Price JL: Architectonic subdivision of the human orbital and medial prefrontal cortex. J Comp Neurol 2003, 460:425 449.

27. Roy AK, Shehzad Z, Margulies DS, Kelly AMC, Uddin LQ, Gotimer K, Biswal BB, Castellanos FX, Milham MP: Functional connectivity of the human amygdala using resting state fMRI. Neuroimage 2009, 45:614 626.

28. Paulus MP, Stein MB: An insular view of anxiety. Biol Psychiatry 2006, 60:383 387.

29. Paulus MP, Stein MB: Interoception in anxiety and depression. Brain Struct Funct 2010, 214:451 463

\section{doi:10.1186/s13587-014-0014-5}

Cite this article as: Klumpp et al:: Resting state amygdala-prefronta connectivity predicts symptom change after cognitive behavioral therapy in generalized social anxiety disorder. Biology of Mood \& Anxiety Disorders 2014 4:14. 